\title{
Calibration of a Triple Frequency Acoustic Backscatter System
}

\author{
Wei-wei LU, Shan-he Huang, Ren-liang Qian
}

\begin{abstract}
The application of acoustic method to measure suspended particle size and concentration profile has been gaining backscattered signal require an inversion algorithm with known acoustic backscatter system constant. This need a system calibration. Using glass spheres which has regular shape as suspension sediments the calibration can be conducted with known scattering characteristics. The result shows that a triple frequency acoustic backscatter system can be calibrated in such way.
\end{abstract}

Index Terms-Suspended sediments, Acoustic, Backscatter, Calibration.

\section{INTRODUCTION}

Using acoustic technology to obtain suspended particle size profile has gain increasing acceptance. Acoustic method can get suspended sediments information non-intrusively with high temporal and spatial resolution [1], which help the acoustic method get this important role in the practical application.

Extracting suspended sediments information from the backscattered signal need know acoustic backscatter system constant. This need calibration. By measuring beam patterns and sensitivity of transducer, calibration of a triple frequency backscatter system can be conducted [2]. But most of laboratories do not have these specialist instrumentation to measure the sensitivities and beam patterns. An alternative method is producing a homogenous suspensions filled with bed sediments and collecting the backscattered signal in far-field. However, this calibration method includes the suspended sediments scattering characteristics, $\mathrm{K}_{\mathrm{s}}$, which is site specific.Using sediments with regular shape as calibration sediments, the system constant, $\mathrm{K}_{\mathrm{t}}$, can be calculated separately[3]. The scattering characteristics of regular shape sediment or spheres has an accurate expression and the measured values of the scattering properties is hard to calculate and a simple expression is proposed and the results show no significantly difference compared to the accurate expression[4]. In the process of inversion, when the attenuation due to sediments scattering cannot be ignored, the inversion become more complicated.Traditionally, an inversion algorithm with iterative calculation is conducted on the backscattered signal to obtain the concentration profile when the sediments scattering attenuation cannot be ignored.The iterative calculation takes a lot of time to converse.

In this study, we use glass spheres as calibration sediments to produce homogenous suspensions, and their backscattering characteristics is easy to calculate.

Wei-wei Lu, Dept of Underwater Sensing, Zhejiang University, Hangzhou, China, Phone No: (+86) 18362890130 .

Shan-he Huang, Dept of Underwater Sensing, Zhejiang University, Hangzhou, China.

Ren-liang Qian, Dept of Underwater Sensing, Zhejiang University, Hangzhou, China.

\section{PROCEDURE OF CALIBRATION}

\section{A. Sediment tower}

To calibrate a triple frequency acoustic backscatter system and examine our matching algorithm, a series of experiments were conducted in the tower in figure (1). There is an electronic motor at the top of tower, used for driving the mixing plate and the turbulence grid have the same purpose, trying to produce homogenous suspension. And you need to control the electronic motor speed. The mixing chamber is used for mixing the water and sediments. Three different frequency transducer is fixed in the tower, respectively 1.0, 2.0, 3.0 MHz. Before adding sediments, we should keep the tower filled with water for a period of time, in order to eliminate the influence of bubbles. Meanwhile, running the electronic motor to help reduce bubbles. Before the next round of experiments using different sediments, you should clean the tower and repeat the above steps to eliminate the influence of bubbles. The pulse repeating frequency used here is $20 \mathrm{~Hz}$ which aims to eliminate the influence of the echo in the tower. To obtain accurate values for $V_{r m s}$, more backscattered signal should be collected due to the backscattered signal is Rayleigh distribution with a standard error in $V_{r m s}$ given approximately by $1 /(2 \sqrt{n})$, where $\mathrm{n}$ is the number of backscattered signal. In this paper, we capture 500 backscattered signal profiles to achieve $2.2 \%$ accuracy in $V_{r m s}$.

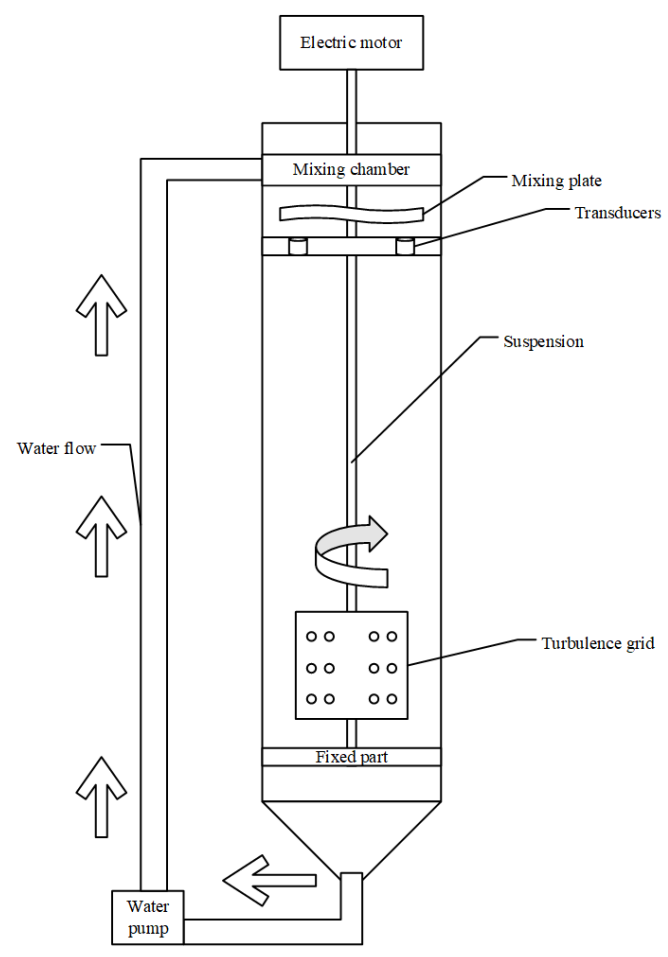

Figure (1): A simple schematic diagram of the experiment tower 


\section{B. Sediments}

Hence, we use glass spheres as our calibration sediments and their shapes are almost spherical. Ideally, the sediments used for calibration should have the same particle size, but it is hard to meet this requirement. The shapes of glass spheres are shown in figure (2). If the sediments have low variance or the particle size distribution is very concentrated, the sediments are also an ideal calibration sediment. The average radius of glass spheres we used here is $111.2,89.5,72.3 \mu \mathrm{m}$.

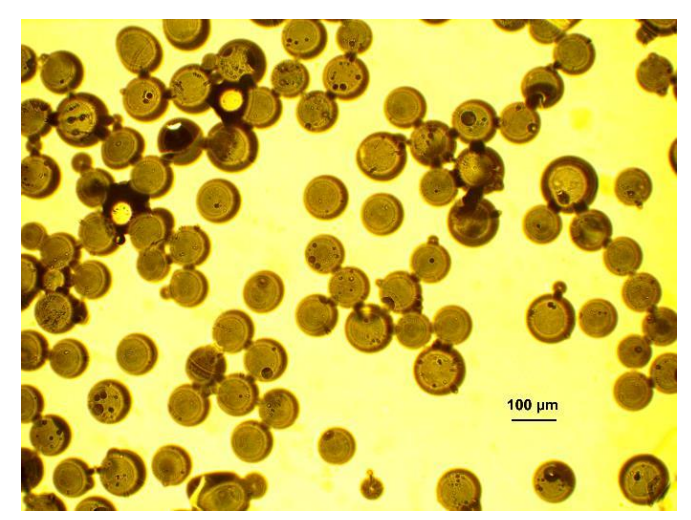

Figure (2): Electron microscope photo graph of Glass spheres

\section{Calibration}

The backscattered signal of different frequency measured in the experiment is an ensemble average over 500 backscattered signal profiles. What's more, the measured range is $0.3-0.7 \mathrm{~m}$ and $0.3 \mathrm{~m}$ is near-field range which is not considered in our experiment. The back scattered signal amplitude decrease along the range due to the attenuation of the water and sediments scattering [5]. After obtaining the $\mathrm{K}_{\mathrm{s}}$ of the glass spheres, we can measure the value of $\mathrm{K}_{\mathrm{t}}$ using equation (1)

$K_{t}=\left(V_{r m s} r / K_{s} M^{1 / 2}\right) e^{2 r\left(\alpha_{w}+\alpha_{s}\right)}$

where

$K_{s}=\frac{f}{\sqrt{a_{0}}}$

$\alpha_{s}=\frac{1}{r} \int_{0}^{r} \zeta M d r$

$\zeta=\frac{3 \chi}{4 a_{0}}$

$\psi=\frac{\left(1+1.35\left(r / r_{n}\right)+\left(2.5\left(r / r_{n}\right)\right)^{3.2}\right.}{1.35\left(r / r_{n}\right)+\left(2.5\left(r / r_{n}\right)\right)^{3.2}}$

with

$\frac{f}{\sqrt{\rho}}=\frac{\left(1-0.25 e^{-((x-1.5) / 0.35)^{2}}\right)\left(1+0.6 e^{-((x-2.9) / 1.15)^{2}}\right) x^{2}}{42+25 x^{2}}$

$\frac{\chi}{\rho}=\frac{0.09 x^{4}}{1380+560 x^{2}+150 x^{4}}$

$r$ is the distance departure from the transducer and $a_{0}$ is the mean particle, $\psi$ is the near-field correction factor, $r_{n}=\pi a_{t}^{2} / \lambda$ is the transducer near-field distance, if $r>r_{n}$, the value $\psi$ equals 1. $a_{t}$ is the transducer radius, $\lambda$ is the wave length of sound, $\alpha_{w}$ accounts for the sound attenuation due to the suspended sediment scattering. $K_{s}$ describes the suspended sediments scattering characteristic. $\rho$ represents the suspended sediments mass density. $f$ describes single sediment backsacttering characteristic and $\chi$ quantifies the degree scattering to surrounding area, which is proportional to scattering attenuation.

The results of calibration experiment are shown in figure (3), the value of $K_{t}$ shows no obvious trend with range.
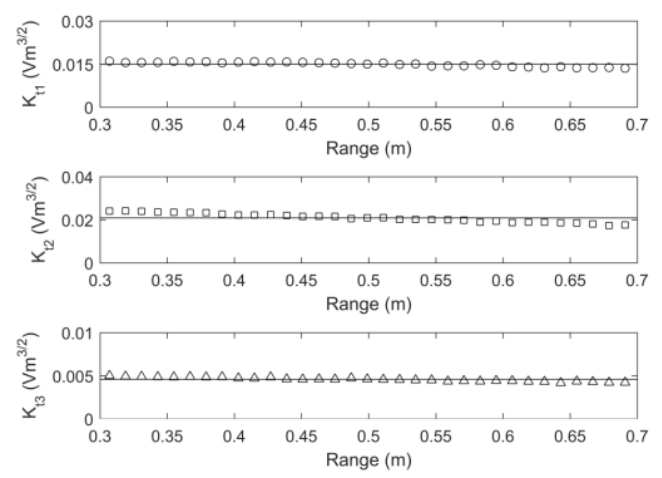

Figure (3): Meared $K_{t}$ with range of different frequency sound, 1.0MHz $\left(K_{\mathrm{t} 1}\right)$, 2.0MHz $\left(\mathrm{K}_{\mathrm{t} 2}\right)$, 3.0MHz $\left(\mathrm{K}_{\mathrm{t} 3}\right)$

In theory, $\mathrm{K}_{\mathrm{t}}$ is also independent of suspension concentration. Here, we conduct a series of experiments on the suspension concentration from 0.15 to $0.80 \mathrm{~g} / \mathrm{L}$ and figure (4) shows the result of $\mathrm{K}_{\mathrm{t}}$ with different suspension concentration. The value of $\mathrm{K}_{\mathrm{t}}$ shows no obvious trend with suspension concentration.
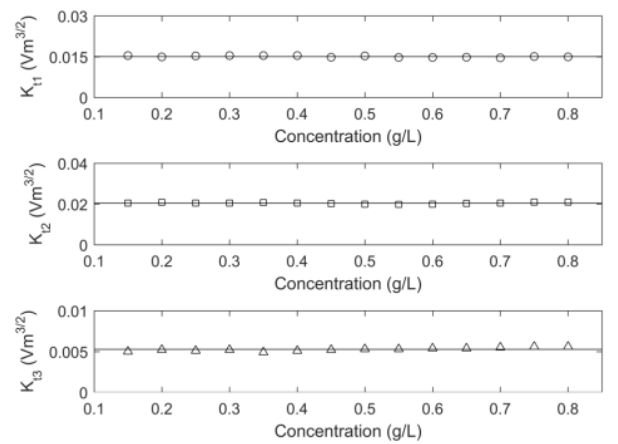

Figure (4): Measured $K_{t}$ with concentration of different frequency sound, $1.0 \mathrm{MHz}\left(\mathrm{K}_{\mathrm{t} 1}\right), 2 \mathrm{MHz}\left(\mathrm{K}_{\mathrm{t} 2}\right), 3.0 \mathrm{MHz}\left(\mathrm{K}_{\mathrm{t} 3}\right)$

The departure of values in figure (4) is caused by experimental error. We also examine the relationship between $\mathrm{K}_{\mathrm{t}}$ and particle size. The results show that the value of $K_{t}$ is independent of particle size.

\section{CONCLUSION}

Using the glass spheres can easily calibrate a triple frequency acoustic backscatter system. And the system constant $\mathrm{K}_{\mathrm{t}}$ shows no trends with the range, the suspension concentration and the particle size.

\section{REFERENCES}

[1] Thorne P D, Agrawal Y C, Cacchione D A. A Comparison of Near-Bed Acoustic Backscatter and Laser Diffraction Measurements of Suspended Sediments[J].IEEE Journal of Oceanic Engineering, 2007, 32(1):225-235.W.-K. Chen, Linear Networks and Systems (Book style). Belmont, CA: Wadsworth, 1993, pp. 123-135.

[2] Hay A E, Wilson D J. Rotary sidescan images of nearshore bedform evolution during a storm[J]. Marine Geology, 1994, 119(1-2):57-65.

[3] Thorne P D, Holdaway G P, Hardcastle P J. Constraining acoustic backscatter estimates of suspended sediment concentration profiles using the bed echo[J]. Journal of the Acoustical Society of America, 1995, 98(4):2280-2288. 
[4] Thorne P D, Hardcastle P J. Acoustic measurements of suspended sediments in turbulent currents and comparison with in-situ samples[J]. The Journal of the Acoustical Society of America, 1997, 101(5): 2603-2614.

[5] Thorne P D, Buckingham M J. Measurements of scattering by suspensions of irregularly shaped sand particles and comparison with a single parameter modified sphere model[J]. Journal of the Acoustical Society of America, 2004, 116(5):2876-2889.

Wei-wei Lu obtained his M.Sc in ocean engineering from Zhejiang university in the year 2020. He is currently working as an engineer in the OPPO company, Guangdong, China. His research includes Suspended Suspension Acoustic Measuring, Signal Processing, Acoustic Instrument Design. He has published one paper in an International Journal.

Shan-he Huang obtained his Ph.D in Naval Architecture and Ocean Engineering in the year 2015. He is currently working as a Professor in the department of Underwater Acoustic Sensing, Zhejiang university, Hangzhou, China. His research interest includes Statistical Signal processing, Data Analysis, Underwater Acoustic Sensing. He has published 15 research papers in International Journals.

Ren-liang Qian obtained his M.Sc in ocean engineering from Zhejiang university in the year 2019. He is currently working as an engineer in the Buy Together Internet company, Shanghai, China. His research includes Suspended Suspension Acoustic Measuring, Computer Vision, Acoustic Instrument Design. He has published two paper in an International Journal. 